\title{
Unionsrechtliche Vorgaben für die Arbeitnehmerfreizügigkeit
}

\author{
Ulrich Becker
}

A. Einleitung $\quad 65$

B. Eigenheit der Arbeitnehmerfreizügigkeit $\quad 67$

C. Voraussetzungen der Arbeitnehmerfreizügigkeit 68

D. Inhalt der Rechte auf und im Aufenthalt 70

E. Grenzen der Arbeitnehmerfreizügigkeit 72

F. Schlußwort $\quad 74$

\section{A. Einleitung}

Nach dem Fall der Berliner Mauer und der Auflösung der politischen Nachkriegsblöcke in Europa standen nicht nur die mittel- und osteuropäischen Staaten vor einer Neuordnung, sondern auch die Europäische Union. Schnell war klar, daß es einen zweiten Europäischen Wirtschaftsraum nicht geben sollte, sondern die Grenzen der europäischen Integration neu zu ziehen waren. Jedoch weckte die politisch gewollte und erforderliche Erweiterung der Europäischen Union angesichts deren Größe und der zumindest zu Beginn bestehenden wirtschaftlichen Unterschiede nicht nur Hoffnungen, sondern auch Befürchtungen. Expertisen wurden geschrieben: über die Folgen einer Arbeitskräftewanderung für die in den alten Mitgliedstaaten bestehenden sozialen Sicherungssysteme ${ }^{1}$ ebenso wie über die europarechtliche Zulässigkeit der Beschränkung der Freizügigkeit ${ }^{2}$. Man entschied sich für Übergangsregelungen ${ }^{3}$, die bei früheren Beitrit-

1 Vgl. Sinn, Die Osterweiterung der EU und die Zukunft des Sozialstaates, in: Leibfried/Wagschal (Hrsg.), Der deutsche Sozialstaat, Bilanzen - Reformen - Perspektiven, 2000, S. 474, 477 ff.; ders., Introduction, in: Social Union and Migration, CESifo No. 3/2004, S. 4, 5.

2 Becker, EU-Erweiterung und differenzierte Integration, 1999.

3 Art. 24 i.V.m. Anhang XII der Akte über die Bedingungen des Beitritts der Tschechischen Republik, der Republik Estland, der Republik Zypern, der Republik Lettland, der Republik Litauen, der Republik Ungarn, der Republik Malta, der Republik Polen, der Republik Slowenien und der Slo- 
ten neuer Mitgliedstaaten schon erprobt worden waren. Ob diese Regelungen, die $\mathrm{zu}$ einigen Auslegungsschwierigkeiten führten, ${ }^{4}$ gelungen und sinnvoll waren oder nicht: Sie sind mit der Herstellung der vollen Arbeitnehmerfreizügigkeit am 1.5.20115 auch im Verhältnis zwischen Polen und Deutschland Vergangenheit.

Damit rückt die Vision Jean Monnets, nach der die europäische Integration nicht der Zusammenführung von Staaten, sondern von Menschen dient, ${ }^{6}$ einen großen Schritt näher. Das paßt zur Neufassung der Ziele der Europäischen Union durch den Lissabonner Vertrag. In Art. 3 EUV ist unmittelbar nach der Förderung von Frieden, Werten und Wohlergehen (Abs. 1) als erste konkrete Aufgabe genannt, daß die Union,,ihren Bürgerinnen und Bürgern einen Raum der Freiheit, der Sicherheit und des Rechts ohne Binnengrenzen“" bietet (Abs. 2).

Die Freizügigkeit gewinnt damit eine eigenständige Bedeutung und emanzipiert sich von einer wirtschaftlich-funktionalen Begründung, wenn auch gerade für die Freizügigkeit der Arbeitnehmer der Zusammenhang zwischen Ortsveränderung und Erwerbstätigkeit bestehen bleibt. Für eine organische Betrachtung hatte im Übrigen schon im Jahr 1819 Friedrich List ein schönes Bild gefunden, als er bemerkte: ,... Zoll und Mautlinien ... lähmen den Verkehr im Innern und bringen ungefähr dieselbe Wirkung hervor, wie wenn jedes Glied des menschlichen Körpers unterbunden wird, damit das Blut ja nicht in ein anderes überfließe"7.

Nun ist also der grenzüberschreitende Fluß von Arbeitskräften auch über die Oder hinweg möglich. Was genau sind dafür die unionsrechtlichen Vorgaben? Wer genießt die Arbeitnehmerfreizügigkeit, was ist deren Inhalt, und was sind deren Grenzen? Diesen Fragen soll im Folgenden nachgegangen werden, in knapper und allgemeiner Form, um eine Kulisse für die nachfolgenden Betrachtungen zum intra-unional-transnationalen Arbeits- und Sozialrecht zu bilden.

wakischen Republik zur Europäischen Union (ABl. 2003 L 236, S. 40); vgl. auch P. Becker, Die deutsche Europapolitik und die Osterweiterung der Europäischen Union, 2011, S. 75.

4 Ausf. dazu Maiß, Die Entsendung von Arbeitnehmern aus den MOE-Staaten auf Werkvertragsbasis nach der EU-Osterweiterung, Eine Untersuchung der grenzüberschreitenden Beschäftigungsmöglichkeiten von Staatsangehörigen aus den Beitrittsstaaten - insbesondere im Baugewerbe - im Kontext der Übergangsbestimmungen zur Arbeitnehmerfreizügigkeit, der Dienstleistungsfreiheit und des Niederlassungsrechts, 2008, S. 223 ff.; vgl. auch Bericht über die Anwendung der im Beitrittsvertrag 2003 festgelegten Übergangsregelungen, $\operatorname{KOM(2006)} 48$ endg.; ferner Bericht über die Auswirkungen, KOM(2008) 765 endg.

5 Vgl. Pressemitteilung IP/11/506 v. 28.4.2011, http://europa.eu/rapid/pressReleasesAction.do? reference $=\mathrm{IP} / 11 / 506 \&$ format $=$ HTML\&aged $=0$ \&language $=$ DE\& guiLanguage $=$ en.

6 Monnet, Les Etats-Unis d'Europe ont commencé, 1955, S. 132: „Nous ne coalisons pas des états, nous unissons des hommes".

7 Verhandlungen der Ständeversammlung des Großherzogthums Baden, Enthaltend die Protokolle der ersten Kammer mit deren Beilagen von ihr selbst amtlich herausgegeben, Müllerscher Hofbuchhandlungs-Verlag, 1819, S. 57. 


\section{B. Eigenheit der Arbeitnehmerfreizügigkeit}

Zunächst ist der Hinweis wichtig, daß es mehrere rechtliche Grundlagen für einen freien Personenverkehr in der Europäischen Union gibt, und daß ganz allgemein von Freizügigkeit zu sprechen ist, wenn sich eine Person von einem Ort zu einem anderen begibt, wobei die Ortsveränderung im Sinne des EU-Rechts ein grenzüberschreitendes Element ist, also von einem Mitgliedstaat in einen anderen erfolgen muß. Freizügigkeit meint dabei das Recht, in einen anderen Mitgliedstaat einzureisen und sich dort aufzuhalten.

Dieses Recht genießen mittlerweile alle Unionsbürger, und zwar auf der Grundlage von Art. 21 AEUV. Weil der EuGH dieser Vorschrift eine sog. direkte Wirkung beimißt, können sich darauf die Unionsbürger berufen: Sie haben ein Recht auf Freizügigkeit. Das hat große Bedeutung für verschiedenste Fragen, wie der zunehmende Umfang der Rechtsprechung zeigt, soll aber hier nicht näher verfolgt werden. Denn es gilt im Unionsrecht nach wie vor der Grundsatz, daß die erwerbsbezogene Freizügigkeit gegenüber der allgemeinen Bürgerfreizügigkeit spezieller ist und deshalb Vorrang besitzt.

Aber auch im Zusammenhang mit einer Erwerbstätigkeit sind verschiedene Varianten der Freizügigkeit zu unterscheiden: Zunächst diejenigen im Zusammenhang mit der Dienstleistungsfreiheit (Art. 56, 57 AEUV), bei der entweder die Dienstleistenden (sog. aktive Dienstleistungsfreiheit) oder die Leistungsempfänger (sog. passive Dienstleistungsfreiheit) wandern können. Will sich ein Leistungserbringer allerdings auf Dauer in einem anderen Mitgliedstaat wirtschaftlich betätigen, dann muß er, und das ist zugleich die dritte Variante, die Niederlassungsfreiheit (Art. 49 AEUV) in Anspruch nehmen. Eine Niederlassung setzt eine ständige Präsenz voraus, die zum Zweck hat, in „stabiler und kontinuierlicher Weise am Wirtschaftsleben“ in einem „Aufnahmemitgliedstaat teilzunehmen". 8

Den drei genannten Varianten ist gemeinsam, daß ihnen eine selbständige Erwerbstätigkeit zugrunde liegt. Das unterscheidet sie zugleich von der Arbeitnehmerfreizügigkeit (Art. 45 AEUV), weil Arbeitnehmer unselbständig tätig werden. Eine wichtige Verbindung zwischen selbständiger und unselbständiger Erwerbstätigkeit stellt die Erbringung von Dienstleistungen unter Mitnahme von Arbeitnehmern dar. Schon vor Jahren hat der Gerichtshof in zwei grundlegenden Entscheidungen, und zwar in den Sachen Rush Portugues $a^{9}$ und Vander Elst ${ }^{10}$, festgestellt, die Freiheit, sich in einen anderen Mitgliedstaat zur Leistungserbringung zu begeben, umfasse auch die Freiheit, die für die Leistungserbringung benötigten Arbeitskräfte mitzunehmen. ${ }^{11}$ Dementsprechend

8 Zuletzt EuGH v. 5.9.2011, Rs. C-347/09 (Dickinger und Ömer), n.v., Rdnr. 35.

9 EuGH v. 27.3.1990, Rs. C-113/89 (Rush Portuguesa), Slg. 1990, I-1417.

10 EuGH v. 9.8.1994, Rs. C-43/93 (Vander Elst), Slg. 1994, I-3803.

11 Wobei Mitnahme nicht im technischen Sinne zu verstehen ist, sondern nur den Bezug zur Dienstleistung zum Ausdruck bringen soll; praktisch kommt vor allem natürlich auch eine Entsendung in 
darf grundsätzlich weder die Einreise noch die Arbeitsaufnahme des Personals durch mitgliedstaatliche Maßnahmen behindert werden. ${ }^{12}$ Damit werden praktisch die Rechte auf Einreise und vorübergehenden Aufenthalt auf die zur Durchführung der Dienstleistung benötigten Arbeitnehmer erstreckt und um das Recht dieser Arbeitnehmer auf ungehinderten Zugang zur Tätigkeit erweitert. Kerngedanke dieser Rechtsprechung ist, wie allgemein bei den Beschränkungsverboten mit Bezug auf die Grenzüberschreitung durch den Dienstleistenden, daß an einen vorübergehend in einem Mitgliedstaat Tätigen nicht dieselben Anforderungen gestellt werden dürfen wie an einen dort Niedergelassenen, sofern der Dienstleistungsfreiheit ihre praktische Wirksamkeit nicht genommen werden soll. ${ }^{13}$ Man könnte hier von einer mittelbaren oder sekundären, durch die Dienstleistungsfreiheit des Arbeitgebers vermittelten Arbeitnehmerfreizügigkeit sprechen. Wir sind damit im Bereich der Entsendungsfälle, die gerade für das Arbeits- und Sozialrecht eine wichtige Rolle spielen. ${ }^{14}$

\section{Voraussetzungen der Arbeitnehmerfreizügigkeit}

Es wurde schon gesagt, daß die Arbeitnehmerfreizügigkeit für unselbständige Tätigkeiten gilt. Der EuGH hat sich bei der Auslegung des unionsrechtlichen Begriffs großzügig gezeigt. Danach sind Arbeitnehmer Personen, ${ }^{15}$ die eine nicht nur völlig untergeordnete und deshalb unwesentliche Tätigkeit ausüben, ${ }^{16}$ wobei diese dadurch charakterisiert sein muß, daß während einer bestimmten Zeit für einen anderen nach dessen

den Leistungsstaat in Betracht, sofern die Arbeitnehmer nicht ihrerseits nur Subunternehmer und damit selbständig tätig sind. Vgl. jetzt auch EuGH v. 10.2.2011, Rs. C-307/09 (Vicoplus), n.v., Rdnr. $31 \mathrm{ff}$.

12 EuGH v. 27.3.1990, Rs. C-113/89 (Rush Portuguesa), Slg. 1990, I-1417, Rdnr. 12: „Infolgedessen hindern die Artikel 59 und 60 EWG-Vertrag einen Mitgliedstaat daran, es einem in einem anderen Mitgliedstaat ansässigen Erbringer von Dienstleistungen zu verbieten, mit seinem gesamten Personal frei in das Gebiet des erstgenannten Staates einzureisen, oder die Einreise des betroffenen Personals von einschränkenden Bedingungen wie der Bedingung der Einstellung von Personal an Ort und Stelle oder der Pflicht zur Einholung einer Arbeitserlaubnis abhängig zu machen. Durch die Auferlegung solcher Bedingungen wird nämlich der Leistungserbringer aus einem anderen Mitgliedstaat gegenüber seinen im Aufnahmeland ansässigen Konkurrenten, die sich ihres eigenen Personals ungehindert bedienen können, diskriminiert und seine Fähigkeit, die Leistung zu erbringen, beeinträchtigt."

13 Allgemein EuGH v. 25.7.1991, Rs. C-76/90 (Säger), Slg. 1991, I-4221, Rdnr. 13.

14 Zur Regelung der Transnationalisierung der Arbeitsverhältnisse wurde, gestützt auf Art. 57 Abs. 2, 66 EGV a.F., die RL 96/71 über die Entsendung von Arbeitnehmern im Rahmen der Erbringung von Dienstleistungen (ABl. 1997 L 18, S. 1) erlassen.

15 Die folgenden Ausführungen sind zum Teil entnommen aus Becker, Arbeitnehmerfreizügigkeit, in: Ehlers (Hrsg.), Europäische Grundrechte und Grundfreiheiten, 3. Aufl. 2009, § 9 Rdnr. 6 ff., wo Voraussetzungen und Inhalte der Arbeitnehmerfreizügigkeit näher dargestellt werden.

Vgl. EuGH v. 23.3.1982, Rs. 53/81 (Levin), Slg. 1982, 1035, Rdnr. 17. 
Weisungen Leistungen erbracht werden, für die als Gegenleistung eine Vergütung gezahlt wird. ${ }^{17}$ Auf weitere Voraussetzungen, insbesondere die Dauer der Erwerbstätigkeit, kommt es nicht an. ${ }^{18}$ Auch Teilzeittätigkeiten ${ }^{19}$ und geringfügige Beschäftigungen $^{20}$ sind als Arbeitnehmertätigkeiten anzusehen. $\mathrm{Zu}$ beachten ist lediglich die Bereichsausnahme in Art. 45 Abs. 4 AEUV für die öffentliche Verwaltung, die aber eng ausgelegt wird.

Freizügigkeitsberechtigt sind die Unionsbürger, also die Staatsangehörigen eines Mitgliedstaates $^{21}$ - während die Arbeitnehmermitnahme darüber hinaus ebenfalls Drittstaatsangehörigen zugute kommen kann. Gemäß der RL 2004/3822 dürfen auch Familienangehörige von Arbeitnehmern 23 in den Beschäftigungsstaat einreisen und dort ihren Wohnsitz nehmen. ${ }^{24}$ Neben diesem abgeleiteten Aufenthaltsrecht räumt Art. 23 RL 2004/38 den Familienangehörigen ein eigenes Recht auf Ausübung irgendeiner Tätigkeit im Lohn- oder Gehaltsverhältnis im gesamten Hoheitsgebiet des betreffenden Mitgliedstaates ein. 25

17 EuGH v. 3.7.1986, Rs. 66/85 (Lawrie-Blum), Slg. 1986, 2121, Rdnr. 17. Ob unentgeltliche Tätigkeiten einbezogen sind, wenn es um Beschäftigungsverhältnisse zu Ausbildungszwecken geht, erscheint fraglich, ließe sich aber mit einem allgemeinen Arbeitnehmerbegriff durchaus vereinbaren, vgl. nur Wölker/Grill, in: v.d. Groeben/Schwarze (Hrsg.), EU-/EG-Vertrag, Bd. 1, 6. Aufl. 2003, vor Art 39-41 EGV, Rdnr. 28 m.w.N.

18 Vgl. EuGH v. 21.6.1988, Rs. 197/86 (Brown), Slg. 1988, 3205, LS 3.

19 Ohne daß die Erzielung eines Mindesteinkommen vorausgesetzt würde, vgl. dazu EuGH v. 23.3.1982, Rs. 53/81 (Levin), Slg. 1982, 1035, Rdnr. 16; zur Abgrenzung gegenüber einer unwesentlichen Tätigkeit EuGH v. 26.2.1992, Rs. C-357/89 (Raulin), Slg. 1992, I-1027, Rdnr. 13.

20 Vgl. dazu EuGH v. 14.12.1995, Rs. C-444/93 (Megner u. Scheffel), Slg. 1995, I-4741, Rdnr. 20.

21 Einschließlich der Staatsangehörigen von EWR-Staaten und der Schweiz, vgl. Becker, in: Ehlers, Europäische Grundrechte und Grundfreiheiten (Fußn. 15), § 9 Rdnr. 30. Zur Gleichbehandlung im Verhältnis EU/Schweiz EuGH v. 6.10.2011, Rs. C-506/10 (Graf und Engel), n.v.

22 RL v. 29.4.2004 über das Recht der Unionsbürger und ihrer Familienangehörigen, sich im Hoheitsgebiet der Mitgliedstaaten frei zu bewegen und aufzuhalten, zur Änderung der Verordnung (EWG) Nr. 1612/68 und zur Aufhebung der Richtlinien 64/221/EWG, 68/360/EWG, 72/194/EWG, 73/148/EWG, 75/34/EWG, 75/35/EWG, 90/364/EWG, 90/365/EWG und 93/96/EWG (AB1. 2004 L 158, S. 77).

23 Zum Begriff vgl. Art. 2 Nr. 2 RL 2004/38.

24 Art. 5 und 7 Abs. 2 RL 2004/38. Das Erfordernis einer ständigen Familienwohnung besteht nicht, vgl. EuGH v. 13.2.1985, Rs. $267 / 83$ (Diatta), Slg. 1985, 567, Rdnr. 17 f. Ob die Freizügigkeit der Angehörigen auch unmittelbar aus Art. 45 AEUV ableitbar ist, erscheint fraglich; richtigerweise müsste für die Begründung eigener Rechte zumindest zusätzlich auf den Schutz durch ein Grundrecht rekurriert werden, vgl. aber auch Wölker/Grill, in: v.d. Groeben/Schwarze, EU-/EG-Vertrag (Fußn. 17), vor Art 39-41 EGV Rdnr. 45 ff.

25 „Der Ehegatte eines Staatsangehörigen eines Mitgliedstaats, der im Hoheitsgebiet eines Mitgliedstaats eine Tätigkeit im Lohn- oder Gehaltsverhältnis oder eine selbständige Tätigkeit ausübt, sowie die Kinder dieses Staatsangehörigen, die noch nicht 21 Jahre alt sind oder denen er Unterhalt gewährt, haben, selbst wenn sie nicht die Staatsangehörigkeit eines Mitgliedstaats besitzen, das Recht, im gesamten Hoheitsgebiet dieses Mitgliedstaats irgendeine Tätigkeit im Lohn- oder Gehaltsverhältnis auszuüben.“ 


\section{Inhalt der Rechte auf und im Aufenthalt}

Art. 45 Abs. 3 AEUV enthält drei grundlegende Rechte, die eine zeitliche Abfolge nachzeichnen. Zunächst gewährt er Arbeitnehmern ausdrücklich das Recht, sich um tatsächlich angebotene Stellen zu bewerben. Garantiert wird damit der freie Zugang zu einer Beschäftigung. ${ }^{26}$ Das umfaßt ein Einreise- und Aufenthaltsrecht und das Recht auf Gleichbehandlung bei der Stellenbewerbung ${ }^{27}$, ohne daß die Einreise im Hinblick auf bestimmte, bereits ausgeschriebene Stellenangebote erfolgen müßte. Diese Rechte bestehen nach der sekundärrechtlichen Ausgestaltung mindestens für drei Monate. ${ }^{28}$

Als zweites garantiert wird das Recht, in einem anderen Mitgliedstaat zu arbeiten und sich dafür dort aufzuhalten. Dazu kommt drittens das Recht, nach Beendigung des Arbeitsverhältnisses im Beschäftigungsstaat zu verbleiben. Der grundlegende Gedanke ist, Beschäftigten zu ermöglichen, daß sie nach Ausscheiden aus dem Arbeitsleben in ihrem gewohnten Umfeld leben. Dementsprechend knüpft das Verbleiberecht an eine Aufgabe der Beschäftigung wegen Erreichens des Rentenalters oder Eintritts einer Erwerbsunfähigkeit an und setzt eine vorangegangene Beschäftigung von einer bestimmten Dauer voraus. ${ }^{29}$ Wird ein Wanderarbeitnehmer arbeitslos, so darf er im Hinblick auf seine berufliche Wiedereingliederung nicht anders behandelt werden als inländische Arbeitnehmer in vergleichbarer Lage. ${ }^{30}$ Seine Einstufung als Arbeitnehmer und insbesondere die Dauer seines weiteren Aufenthaltsrechts hängen von den Umständen ab und sind auch sekundärrechtlich nicht eindeutig geregelt. ${ }^{31} \mathrm{Zu}$ erwähnen ist aber eine Er-

26 In diesem Zusammenhang spielt die Anerkennung von beruflichen Fähigkeiten und der darüber vorhandenen Nachweise eine große Rolle, vgl. dazu RL 2005/36/EG (ABl. 2005 L 255, S. 22), die RL 89/48 (AB1. 1989 L 19, S. 16) und RL 92/51 (ABl. 1992 L 209, S. 25) samt sektoriellen Ergänzungsrichtlinien Ende 2007 abgelöst hat; zu den reglementierten Berufen i.S.v. RL 89/48 zuletzt EuGH v. 22.12.2010, Rs. C-118/09 (Koller), n.v., und v. 5.4.2011, Rs. C-424/09 (Toki), n.v. Dazu, daß ein solcher Nachweis gefordert werden kann und die Anforderungen nicht wegen Art. 45 AEUV herabzusetzen sind, bezogen auf den juristischen Vorbereitungsdienst, EuGH v. 10.12.2009, Rs. C-345/08 (Pesla), Slg. 2009, I-11677.

27 Eine Bewerbung kann aber in der Regel nur Erfolg haben, wenn eine entsprechende Qualifikation erfüllt wird. Deshalb ist mit der RL 2005/36/EG nun auch die Anerkennung von im europäischen Ausland erworbenen Qualifikationen und Zeugnissen für reglementierte Berufe festgesetzt worden. Siehe dazu Englmann/Müller, Brain Waste, Die Anerkennung von ausländischen Qualifikationen in Deutschland, S. 34 ff. (abrufbar unter: http://www.berufliche-anerkennung.de/brain\%20waste.pdf).

28 Nach Art. 6 Abs. 1 der RL 2004/38 steht jedem Unionsbürger ein Aufenthaltsrecht für die Dauer von höchstens 3 Monaten zu bzw. nach Art. 7 Abs. 1 lit. b der RL bei Vorliegen ausreichender Existenzmittel und umfassendem Krankenversicherungsschutz auch darüber hinaus.

29 Vgl. näher Art. 17 RL 2004/38.

30 Art. 7 Abs. 1 VO 492/2011 über die Freizügigkeit der Arbeitnehmer innerhalb der Union (ABl. 2011 L 141, S. 1).

31 Einzelheiten sind umstritten, insbesondere auch, ob zwischen freiwilliger und unfreiwilliger Arbeitslosigkeit zu differenzieren ist; vgl. näher Randelzhofer/Forsthoff, in: Grabitz/Hilf (Hrsg.), Recht der EU, Art. 39 EGV (Stand 2011), Rdnr. 45 ff.; vgl. zur aufenthaltsrechtlichen Stellung bei unfreiwilliger Arbeitslosigkeit Art. 7 Abs. 3 RL 2004/38. 
weiterung des Verbleiberechts durch die Rechtsprechung. Der EuGH hat aus dem Umstand, daß ausländische Arbeitnehmer ein Recht auf Zugang zu Berufsschulen und Umschulungseinrichtungen besitzen, ${ }^{32}$ geschlossen, auch Studenten könnten ausnahmsweise die Vergünstigungen für Arbeitnehmer in Anspruch nehmen, wenn zwischen dem Studium und einer zuvor ausgeübten Berufstätigkeit ein Zusammenhang besteht. ${ }^{33}$ Das sichert, grundsätzlich unabhängig von der Dauer der Beschäftigung, ${ }^{34}$ ein Aufenthaltsrecht und ein Recht auf Förderung der Hochschulausbildung.

Mit diesen Kernrechten erschöpfen sich die Rechte von unionsangehörigen Arbeitnehmern keineswegs. Auch im Zusammenhang mit der Arbeitnehmerfreizügigkeit gilt das Diskriminierungsverbot, besonders geregelt in Art. 45 Abs. 2 AEUV. Dieses Diskriminierungsverbot, das sich auch auf Tarif- und Einzelverträge bezieht, ${ }^{35}$ umfaßt jeden Aspekt der Berufstätigkeit. Praktisch wichtig ist ferner eine sekundärrechtliche Bestimmung, Art. 7 Abs. 2 VO 492/2011. Sie räumt einen Anspruch auf „die gleichen sozialen und steuerlichen Vergünstigungen wie die inländischen Arbeitnehmer" ein. ${ }^{36} \mathrm{Zu}$ den steuerlichen Vergünstigungen zählen etwa die Abzugsfähigkeit von Ausgaben, ${ }^{37}$ die Steuerrückerstattung 38 oder das Ehegattensplitting ${ }^{39}$, wobei es eine wichtige Rolle spielt, in welchem Staat die Einkünfte besteuert werden. ${ }^{40}$ Der Begriff der sozialen Vergünstigungen wird vom Gerichtshof weit ausgelegt und erfaßt ,alle Vergünstigungen, die - ob sie an einen Arbeitsvertrag anknüpfen oder nicht - den inländischen Arbeitnehmern hauptsächlich wegen ihrer objektiven Arbeitnehmereigenschaft oder einfach wegen ihres Wohnortes im Inland gewährt werden und deren Ausdehnung auf die

32 Art. 7 Abs. 3 VO 492/2011.

33 EuGH v. 21.6.1988, Rs. C-39/86 (Lair), Slg. 1988, 3161, Rdnr. 35 ff; vgl. zur Abgrenzung auch EuGH v. 26.2.1992, Rs. C-357/89 (Raulin), Slg. 1992, I-1027 ff.

34 Allerdings unter dem Vorbehalt, daß Mißbräuche ausgeschlossen sind, EuGH v. 21.6.1988, Rs. C39/86 (Lair), Slg. 1988, 3161, Rdnr. 43 ff.; vgl. auch EuGH v. 21.6.1988, Rs. C-197/86 (Brown), Slg. 1988, 3205, Rdnr. 22 ff.

35 Zur Nichtigkeit entgegenstehender Absprachen Art. 7 Abs. 4 VO 492/2011; zur unmittelbaren Anwendbarkeit der günstigeren Bestimmungen EuGH v. 15.1.1998, Rs. C-15/96 (Schöning), Slg. 1998, I-47 ff.

$36 \mathrm{Ob}$ der Anspruch auch für Arbeitslose und Arbeitsuchende gilt, ist nicht unumstritten, vgl. Randelzhofer/Forsthoff, in: Grabitz/Hilf, Recht der EU (Fußn. 31), Art. 39 EGV Rdnr. 159; soweit der Arbeitnehmerstatus auf diese Personen erstreckt wird, ist die Geltung aber schon zwingende Konsequenz und für eine Differenzierung kein Platz. Im Übrigen verliert die Diskussion durch das allgemeine Freizügigkeitsrecht an Bedeutung. Vgl. ferner Cordewener, Europäische Grundfreiheiten und nationales Steuerrecht, 2002.

37 Vgl. EuGH v. 28.1.1992, Rs. C-204/90 (Bachmann), Slg. 1992, I-249 ff.

38 Vgl. EuGH v. 8.5.1990, Rs. C-175/88 (Biehl), Slg. 1990, I-1779 ff.; EuGH 14.2.1995, Rs. C279/93 (Schumacker), Slg. 1995, I-225 ff.

39 Vgl. EuGH v. 16.5.2000, Rs. C-87/99 (Zurstrassen), Slg. 2000, I-3337 ff.

40 Keineswegs aber wird, weder primär- noch sekundärrechtlich, ein harmonisiertes Steuersystem geschaffen. Insbesondere bleiben die Mitgliedstaaten auch zuständig, die Kriterien für die Besteuerung von Einkünften festzulegen; dazu und zu den Grenzen des Diskriminierungsverbots zuletzt EuGH v. 15.9.2011, Rs. C-240/10 (Schulz-Delzers und Schulz), n.v. 
Arbeitnehmer, die Staatsangehörige eines anderen Mitgliedstaats sind, deshalb als geeignet erscheint, deren Mobilität innerhalb der Gemeinschaft zu erleichtern."41 Einbezogen sind damit etwa Ausbildungsbeihilfen, ${ }^{42}$ Pflegegeld, ${ }^{43}$ Hilfen zum Lebensunterhalt wie die Sozialhilfe oder ein Mindesteinkommen, ${ }^{44}$ Familienbeihilfen einschließlich von Fahrpreisermäßigungen 45 etc. Die Vergünstigungen müssen aber keineswegs in einer Geld- oder Sachleistung, sondern können auch in der Einräumung sonstiger Positionen bestehen; so zählen zu ihnen das Recht zur Nutzung der eigenen Sprache vor Gericht ${ }^{46}$ und das Aufenthaltsrecht für nichteheliche Partner ${ }^{47}$. Ausgenommen bleiben aber staatsbürgerliche und an eine besondere Vorgeschichte im Heimatstaat anknüpfende Rechte. ${ }^{48}$ Gesondert geregelt ist die Teilhabe am Wohnungsmarkt. ${ }^{49}$ Ebenso ist es die soziale Sicherheit, die hier noch angesprochen werden soll und in späteren Beiträgen ${ }^{50}$ ausführlich gewürdigt wird.

\section{E. Grenzen der Arbeitnehmerfreizügigkeit}

$\mathrm{Zu}$ den Grenzen der Arbeitnehmerfreizügigkeit müssen einige Hinweise genügen. Die in Art. 45 Abs. 3 AEUV genannten Rechte können aus „Gründen der öffentlichen Ordnung, Sicherheit und Gesundheit" beschränkt werden, und nichts anderes gilt für das Diskriminierungsverbot, weil die Arbeitnehmerfreizügigkeit als einheitliche Grundfreiheit verstanden werden muß.

Die genannten Schranken sind in erster Linie für die Aufenthaltsrechte von ausländischen Arbeitnehmern von Bedeutung, nämlich wenn es um deren Ausweisung und Abschiebung geht. In dieser Hinsicht enthält das Sekundärrecht, namentlich die schon erwähnte RL 2004/38, Konkretisierungen. Danach bezieht sich der ordre public-

41 Vgl. nur EuGH v. 12.5.1998, Rs. C-85/96 (Martínez Sala), Slg. 1998, I-2691, Rdnr. 25 ff.; nach der Entscheidung ist gegenüber der VO 883/2004 keine Abgrenzung mehr erforderlich.

42 Vgl. EuGH v. 27.9.1988, Rs. C-235/87 (Matteucci), Slg. 1988, 5589 ff.; vgl. zu Überbrückungsbeihilfen Urt. v. 16.9.2004, Rs C-400/02 (Merida), Slg. 2004, I-8471.

43 Vgl. EuGH v. 21.2.2006, Rs. C-286/03 (Hosse), Slg. 2006, I-1771, Rdnr. 20 ff.

44 Vgl. EuGH v. 18.6.1987, Rs. C-316/85 (Lebon), Slg. 1987, 2811 ff.

45 EuGH v. 30.9.1975, Rs. C-32/75 (Cristini), Slg. 1975, 1085 ff.

46 EuGH v. 11.6.1985, Rs. C-137/84 (Mutsch), Slg. 1985, $2681 \mathrm{ff}$.

47 EuGH v. 17.4.1986, Rs. C-59/85 (Reed), Slg. 1986, 1283 ff. Aufenthaltsrechte für ausländische homosexuelle Lebenspartner sind ohne weiteres eingeschlossen, sofern diese Inländern gewährt werden. Die Mitgliedstaaten bleiben aber berechtigt, bei den Voraussetzungen für einen (auch abgeleiteten) Daueraufenthalt nach Staatsangehörigkeit bzw. Aufenthaltsstatus zu differenzieren, vgl. EuGH v. 11.4.2000, Rs. C-356/98 (Kaba), Slg. 2000, I-2623, Rdnr. 30 ff.

48 Wie das Wahlrecht und Kriegsopferleistungen.

49 Art. 9 VO 492/2011.

50 Vgl. die Beiträge unter D. 
Vorbehalt nicht auf ,wirtschaftliche Zwecke“"51, und die Berufung auf die öffentliche Ordnung und Sicherheit setzt ein Anknüpfen an das ,persönliche Verhalten“ des Betroffenen voraus. 52

Zumindest eine Rechtfertigung von Beschränkungen und nur mittelbaren Diskriminierungen ist auch durch die sog. zwingenden Gründe des Allgemeininteresses möglich, allerdings immer unter dem Vorbehalt der Verhältnismäßigkeit. Beispiele aus der Rechtsprechung beziehen sich auf den Schutz vor der mißbräuchlichen Führung akademischer Grade ${ }^{53}$, den Schutz des Sports, insbesondere des sportlichen Wettbewerbs ${ }^{54}$ auch unter Berücksichtigung eines Wettbewerbs unter Nationen, was im Ergebnis zu einem speziellen Schutz für Nationalmannschaften führt. 55 Vor diesem Hintergrund kann sich die Deutsche Fußballnationalmannschaft glücklich schätzen, daß Lukas Podolski und Miroslav Klose trotz ihrer polnischen Wurzeln die Deutsche Staatsangehörigkeit besitzen.

Allgemeinere Schutzgüter sind die Aufrechterhaltung öffentlicher Einrichtungen, etwa funktionierender Steuerrechts- oder Sozialleistungssysteme, ${ }^{56}$ von kollektiven Verhandlungssystemen oder Universitäten ${ }^{57}$, ebenso der Schutz der Arbeitnehmer, der Verbraucher sowie der einer ordnungsgemäßen Berufsausübung 58 .

Ausgeschlossen ist hingegen hier wie bei allen anderen Grundfreiheiten eine Berufung auf praktische Schwierigkeiten ${ }^{59}$ oder eine Verwaltungsvereinfachung 60 , ebenso auf rein wirtschaftliche Gründe. Damit schließt sich der Kreis zu den Übergangsbestimmungen, die im Rahmen der Osterweiterung getroffen worden waren. Denn gerade weil sie allgemeine, nicht für eine Rechtfertigung einzelner Eingriffsmaßnahmen ausreichende Schwierigkeiten erwarteten, hatten die meisten der früheren EG-Staaten darauf gedrängt, die Freizügigkeit erst nach Ablauf einer Übergangsfrist zu verwirklichen.

51 Art. 27 Abs. 1 RL 2004/38.

52 Art. 27 Abs. 2 RL 2004/38. Zur unmittelbaren Anwendbarkeit der letztgenannten Bestimmung nur EuGH v. 4.12.1974, Rs. C-41/74 (van Duyn), Slg. 1974, 1337, Rdnr. 13 ff.; allerdings äußerte sich der EuGH dort zu der RL 64/221, die aber dieselben Vorschriften enthält. Danach genügen insbesondere strafrechtliche Verurteilungen für eine Aufenthaltsbeendigung nicht.

53 EuGH v. 13.1.1993, Rs. C-19/92 (Kraus), Slg. 1993, I-1663 ff.

54 EuGH v. 11.4.2000, Rs. C-51/96 \& C-191/97 (Deliège), Slg. 2000, I-2549; in diesem Zusammenhang zu dem Zweck, die Anwerbung und die Ausbildung junger Spieler zu fördern, EuGH v. 16.3.2010, Rs. C-325/08 (Olympique Lyonnais), Slg. 2010, I-2177.

55 EuGH v. 15.12.1995, Rs. C-415/93 (Bosman), Slg. 1995, I-4921 ff.

56 Vgl. etwa EuGH v. 28.1.1992, Rs. C-204/90 (Bachmann), Slg. 1992, I-249 ff.

57 EuGH v. 2.8.1993, Rs. C-259/91 (Allué II), Slg. 1993, I-4309ff.

58 Zumindest in der Sache verfährt der EuGH hier offensichtlich großzügig, wenn er etwa bei Forderung bestimmter Qualifikationen das geschützte Rechtsgut nicht näher benennt, was allerdings auch daran liegen kann, daß in den entschiedenen Fällen der Eingriff zumeist ohnehin unverhältnismäßig ist, vgl. nur EuGH v. 25.11.1999, Rs. C-281/98 (Angonese), Slg. 2000, I-4139 ff.

59 Vgl. EuGH v. 12.3.1998, Rs. C-187/96 (Kommission/Griechenland), Slg. 1998, I-1095 ff.

60 Offengelassen, aber so angedeutet auch für die Erleichterung der Steuererhebung in EuGH v. 16.5.2000, Rs. C-87/99 (Zurstrassen), Slg. 2000, I-3337, Rdnr. 25 ff. 


\section{F. Schlußwort}

Insgesamt zeigt der geraffte Überblick, daß die Voraussetzungen der Arbeitnehmerfreizügigkeit weit verstanden werden und deren Inhalt stark durch Sekundärrecht geprägt wird. Zudem hat der EuGH gerade bei der Arbeitnehmerfreizügigkeit die Adressaten, also die Verpflichteten, weit gefaßt: Auch Eingriffe durch „kollektive Regelungen im Arbeits- und Dienstleistungsbereich“ können durch Art. 45 AEUV verboten sein 61 , und zumindest das Verbot der Diskriminierung soll in Einzelfällen auch durch den Arbeitgeber zu beachten sein. ${ }^{62}$

Die Arbeitnehmerfreizügigkeit war und ist ein Grundpfeiler der EU, sozusagen die menschliche Seite des Binnenmarkts. Sie ist nun Teil eines umfassenden Freizügigkeitsrechts, das als Bürgerrecht nicht nur die europäische Integration persönlich erfahrbar macht, sondern auch einen wichtigen Zusammenhang zwischen deren Bedeutung und deren Legitimation herstellt. In diesem neuen Rahmen bleibt die Arbeitnehmerfreizügigkeit ein wichtiges Mittel, so abschließend noch einmal Jean Monnet, um eine „Angleichung der Lebens- und Arbeitsbedingungen“" zu erzielen. ${ }^{63}$

61 EuGH v. 12.12.1974, Rs. C-36/74 (Walrave), Slg. 1974, 1405, Rdnr. 16 ff.; EuGH v. 15.12.1995, Rs. C-415/93 (Bosman), Slg. 1995, I-4921, Rdnr. 84 ff.

62 EuGH v. 25.11.1999, Rs. C-281/98 (Angonese), Slg. 2000, I-4139, Rdnr. 30 f., 36 f.

63 Rede vor der Gemeinsamen Versammlung v. 15.6.1953 in Straßburg, zitiert nach: www.europareden.de/veranstaltungen/SS05/schwarz/monnet.pdf (20.11.2011). 


\title{
Legal instruments for the development of the European Labour Market
}

\author{
Dagmara Skupień
}

$\begin{array}{ll}\text { Introduction } & 75\end{array}$

A. Institutional framework $\quad 77$

I. The Advisory Committee $\quad 77$

II. The Technical Committee $\quad 78$

III. European Coordination Office 79

B. EURES $\quad 80$

C. Support of migration of young workers and graduates 82

D. Solvit network

E. Mutual recognition of professional qualifications 86

I. Basic notions $\quad 86$

II. Levels of professional qualifications $\quad 87$

III. Compensatory measures $\quad 88$

$\begin{array}{ll}\text { IV. Procedural rules } & 90\end{array}$

V. ECJ jurisprudence $\quad 90$

Conclusions $\quad 93$

Introduction

The free movement of workers in the EU is supported by instruments which aim at facilitating the transnational mobility of job-seekers. These instruments have the legal basis in Article 46 TFEU (ex. Article 40 TEC) which gives the competence to the European Parliament and the Council to issue directives or make regulations setting out measures required to bring about freedom of movement for workers in particular by ensuring close cooperation between national employment services, by abolishing any administrative procedures, practices or qualifying periods in respect of eligibility for available employment, the maintenance of which would form an obstacle to liberalization of the movement of workers and by setting up appropriate machinery to match the demand and supply of work in the EU. 